\title{
Misdiagnosed spontaneous intracranial hypotension complicated by subdural hematoma following lumbar puncture
}

This article was published in the following Dove Press journal:

International Journal of General Medicine

15 January 2014

Number of times this article has been viewed

\author{
Nissrine Louhab' \\ Nawal Adali' \\ Mehdi Laghmari \\ Wafae El Hymer ${ }^{2}$ \\ Said Ait Ben Ali ${ }^{2}$ \\ Najib Kissani' \\ 'Neurology Department, \\ ${ }^{2}$ Neurosurgery Department, \\ University Hospital of \\ Mohammed the $\mathrm{VI}^{\text {th }}$, \\ Cadi Ayyad University, \\ Marrakech, Morocco
}

Correspondence: Nissrine Louhab Neurology Department, University Hospital of Mohammed the $\mathrm{Vl}^{\text {th }}$, Cadi Ayyad University,

Avenue Prince Moulay Abdellah, BP 5II, 40000 Marrakesh, Morocco Tel +2I2 67457802 I

Email lounissrine@yahoo.fr
Introduction: Spontaneous intracranial hypotension is an infrequent cause of secondary headache due to cerebrospinal fluid (CSF) hypovolemia.

Objective: To describe a case of headache revealing spontaneous intracranial hypotension complicated by subdural hematoma following lumbar puncture.

Observation: A 34-year-old man presented with acute postural headache. The first cerebral computed tomography scan was normal. Lumbar puncture showed hyperproteinorachy at $2 \mathrm{~g} / \mathrm{L}$ with six lymphocytic cells. The headache became very intense. At admission, clinical examination was normal. Ophthalmological examination did not show any abnormalities. Encephalic magnetic resonance imaging (MRI) showed bilateral subdural hematoma with tonsillar descent simulating Chiari type I malformation. After surgical drainage and symptomatic treatment, the patient was discharged with no recurrence.

Conclusion: Spontaneous intracranial hypotension is associated with simple clinical presentation, orthostatic headache, and characteristic MRI findings. Misdiagnosed, it leads to unnecessary procedures.

Keywords: intracranial hypotension, headache, magnetic resonance imaging

\section{Introduction}

Spontaneous or idiopathic intracranial hypotension $(\mathrm{SIH})$ is rare. ${ }^{1}$ Careful history taking and a high level of suspicion are essential to diagnose this syndrome. Brain imaging, especially magnetic resonance imaging (MRI), is very helpful for diagnosis confirmation. Misdiagnosis leads to unnecessary procedures. The authors present this illustrative case of postural headache revealing SIH misdiagnosed and complicated by subdural hematoma following lumbar puncture.

\section{Case report}

A 34-year-old man without any headache history, cardiovascular risk factor, fever, or antecedent trauma had since September 3, 2010 acute postural headache that occurred within 15 minutes of assuming an upright position; it began as a pain over the vertex, and rapidly became holocephalic, with photophobia, nausea, and vomiting. The headache resolved within 10 minutes of assuming the supine position. The patient did not have aura, visual change, or other neurological symptoms. Clinical examination was normal, and did not found any hyperflexibility of skin or joints. The first cerebral computed tomography scan was normal. A brain MRI with angio-sequences did not show cerebral thrombophlebitis, and was interpreted as normal (Figure 1). Lumbar puncture was performed, without a measure of cerebrospinal fluid (CSF) pressure, showed hyperproteinorachy at $2 \mathrm{~g} / \mathrm{L}$, and six 


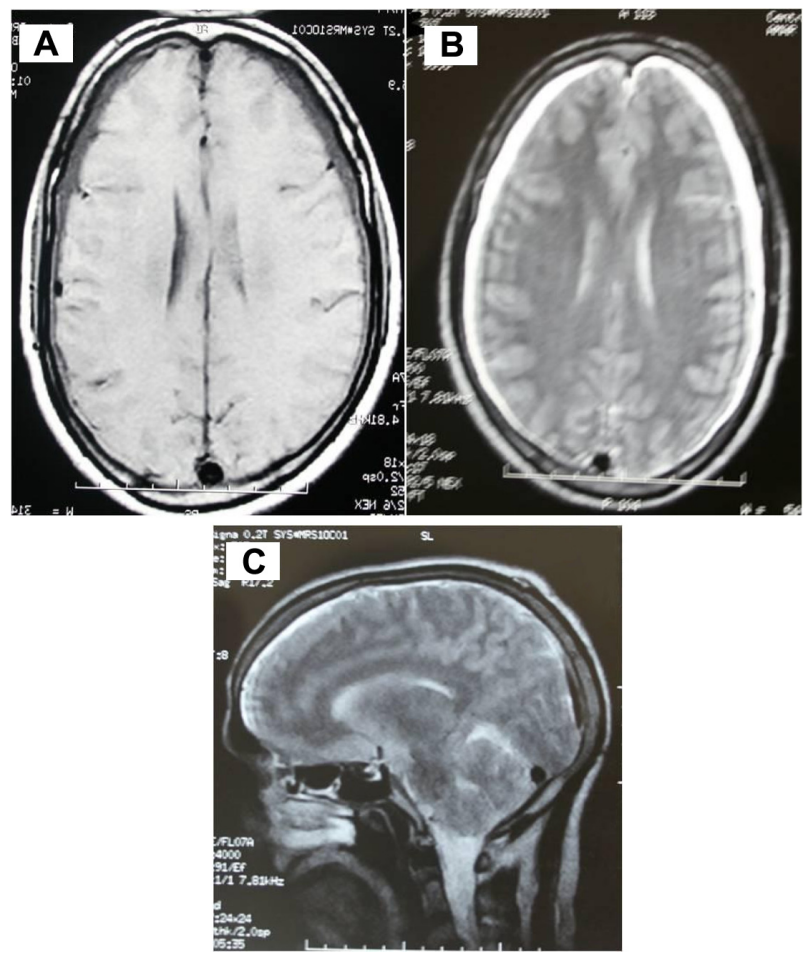

Figure I A and B: Axial cerebral MRI in TI and T2 weighted views, without contrast injection, showing bilateral subdural fluid collection and engorgement of superior sagittal sinus. C: Sagittal T2 weighted views, showing obliteration of prepontine cistern.
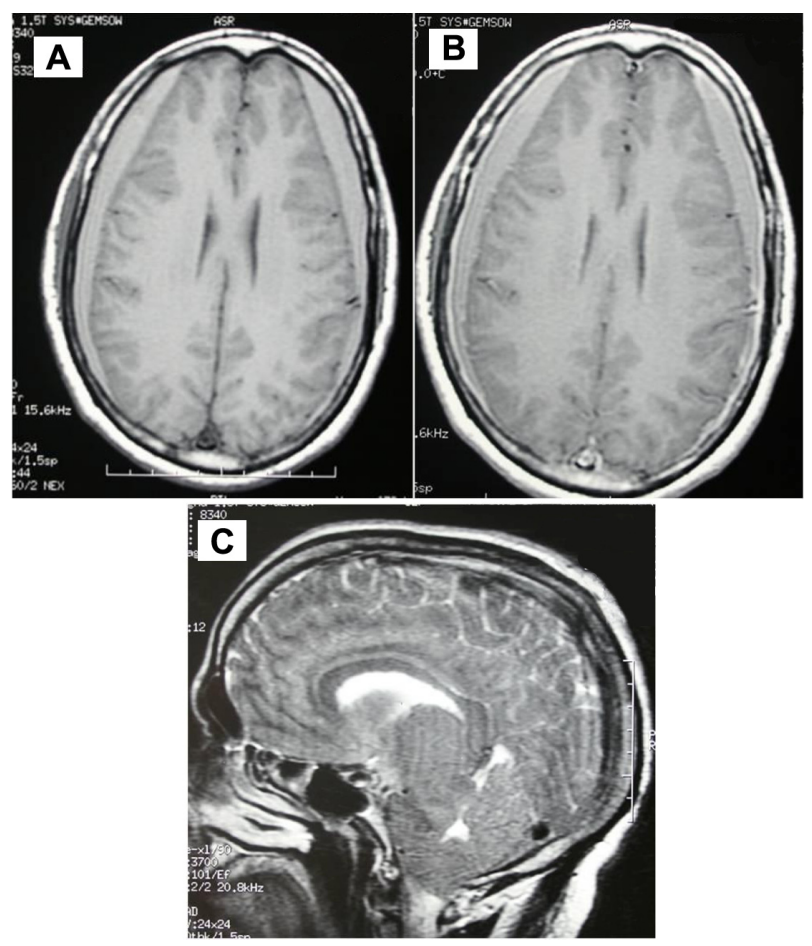

Figure 2 A and B: After lumbar puncture, axial cerebral MRI TI weighted views, with Gadolinum injection, showing bilateral subdural hematoma. C: Sagittal T2 weighted views, showing tonsilar descent simulating Chiari type I malformation. cells with lymphocytosis. The headache became very intense. At admission, clinical examination was normal. Ophthalmological examination did not show any abnormalities. A second brain MRI showed bilateral subdural hematoma with tonsillar descent, simulating Chiari type I malformation (Figure 2). The patient was treated by surgical drainage of the subdural hematoma and bed rest. The blood patch was not realized. The headache was completely resolved 2 days after surgery. There was no recurrence after 3 years of follow-up.

\section{Discussion}

This observation describes a case of postural headache revealing $\mathrm{SIH}$ misdiagnosed and complicated by subdural hematoma following lumbar puncture. In the literature, there are many studies describing SIH, specifying clinical, paraclinical, and therapeutic characteristics. ${ }^{2} \mathrm{SIH}$ is caused by single or multiple spinal CSF leaks. The incidence has been estimated at five per 100,000 per year, with a peak around 40 years of age. Women are more affected, but not exclusively, as shown in our case. ${ }^{3}$ A postural headache is the clinical hallmark of SIH, as seen in this case. It occurs or worsens within 15 minutes after sitting or standing, with at least one of the following: one of neck stiffness, tinnitus, hypacusia, photophobia, or nausea. ${ }^{4}$ Rarely, this condition could happen with a nonpositional headache preceding a typical orthostatic headache. ${ }^{5}$ The diagnosis is confirmed by encephalic MRI and axial and sagittal $\mathrm{T} 1$ sequences, with intravenous administration of gadolinium showing subdural fluid-collection enhancement of the pachymeninges, engorgement of venous structures, pituitary hyperemia, and sagging of the brain., Although typical, these radiological features may be missed, leading to misdiagnosis of this condition. ${ }^{7,8}$

That was the case in this patient, for whom subdural fluid collection was neglected in the first MRI, interpreted as normal (Figure 1), because it did not include gadoliniumenhanced sequences. This may have contributed to the misdiagnosis in this case, as diffuse pachymeningeal enhancement is a very characteristic sign of intracranial hypotension. In some patients with $\mathrm{SIH}$, herniation of the cerebellum and medulla through the foramen magnum occurs simulating Chiari I malformation, similar to this patient's condition. Other investigations (spinal MRI, radioisotope cisternography, and computed tomographic myelography) are indicated to identify the site of CSF leak. ${ }^{5}$ For financial reasons, these explorations were not performed in this case.

CSF constituents are usually normal, although a high protein concentration and lymphocytic pleocytosis may be 
seen, similar to our case, which is probably due to increased permeability of dilated meningeal blood vessels and a decrease of CSF flow in the lumbar subarachnoid space. ${ }^{9}$ In our case, lumbar puncture could be a plausible explanation of bilateral subdural hematoma, which required emergency surgery. Many cases of SIH resolve spontaneously and do not require any specific therapy. ${ }^{5}$ Conservative measures consist of bed rest, oral hydration, generous caffeine intake, and use of an abdominal binder. ${ }^{5}$

The mainstay of treatment is the injection of antilogous blood into the spinal epidural space, the so-called epidural blood patch. ${ }^{10,11}$ Percutaneous placement of fibrin sealant and surgical CSF leak repair can also be performed, but outcomes have been poorly studied.

\section{Conclusion}

$\mathrm{SIH}$ is a unique medical syndrome that can be revealed by orthostatic headache and which has established criteria in the International Classification of Headache Disorders. If not diagnosed, it may lead to unnecessary exhaustive investigations and treatments. The prognosis is excellent if precociously and properly diagnosed.

\section{Disclosure}

The authors report no conflicts of interest in this work.

\section{References}

1. Paldino M, Mogilner AY, Tenner MS. Intracranial hypotension syndrome: a comprehensive review. Neurosurg Focus. 2003;15:ECP2.

2. Roux X, De Greslan T, Sallansonnet-Froment M, et al. Syndrome d'hypotension spontanée du liquide cérébrospinal. Revue de la littérature à propos d'un cas [Spontaneous low cerebrospinal fluid pressure syndrome. A case report and literature review]. La Rev Med Interne. 2008;29:652-657. French.

3. Schievink WI. Misdiagnosis of spontaneous intracranial hypotension. Arch Neurol. 2003;60:1713-1718.

4. Headache Classification Subcommittee of the International Headache Society. The International Classification of Headache Disorders. 2nd ed. Cephalalgia. 2004;24 Suppl 1:1-160.

5. Schievink WI. Spontaneous spinal cerebrospinal fluid leaks and intracranial hypotension. JAMA. 2006;295:2286-2296.

6. Dillon WP, Fishman RA. Some lessons learned about the diagnosis and treatment of spontaneous intracranial hypotension. AJNR Am J Neuroradiol. 1998;19:1001-1002.

7. Sainani NI, Lawande MA, Pungavkar SA, Desai M, Patkar DP, Mohanty PH. Spontaneous intracranial hypotension: a study of six cases with MR findings and literature review. Australas Radiol. 2006;50: 419-423.

8. Trappolini M, Clarice A, Scorza A, et al. A case of spontaneous intracranial hypotension with typical magnetic resonance images. $J$ Headache Pain. 2006;7:44-46.

9. Mokri B. Spontaneous low cerebrospinal pressure/volume headaches. Curr Neurol Neurosci Rep. 2004;4:117-124.

10. Sencakova D, Mokri B, McClelland RL. The efficacy of epidural blood patch in spontaneous CSF leaks. Neurology. 2001;57:1921-1923.

11. Berroir S, Loisel B, Ducros A, et al. Early epidural blood patch in spontaneous intracranial hypotension. Neurology. 2004;63:1950-1951.
International Journal of General Medicine

\section{Publish your work in this journal}

The International Journal of General Medicine is an international, peer-reviewed open-access journal that focuses on general and internal medicine, pathogenesis, epidemiology, diagnosis, monitoring and treatment protocols. The journal is characterized by the rapid reporting of reviews, original research and clinical studies across all disease areas.

\section{Dovepress}

A key focus is the elucidation of disease processes and management protocols resulting in improved outcomes for the patient. The manuscript management system is completely online and includes a very quick and fair peer-review system. Visit http://www.dovepress.com/ testimonials.php to read real quotes from published authors. 